\title{
Current Situation and Development Trend of Policies for Balanced Development of Urban and Rural Education in Eastern, Central and Western China from the Perspective of Educational Equity
}

\section{Jianwei Yang}

The Education University of Hong Kong, 999077

\begin{abstract}
Since the 21st century, China has achieved some success in equity in urban-rural education, and the policies issued have effectively eased the economic pressure of families with special problems and enabled rural education to develop in a sustained and healthy way. In addition, some relevant auxiliary policies have been implemented, providing large amounts of resources for rural education, greatly improving the level of education and teaching and the enthusiasm of rural education. Based on this, this article analyzes the current situation and development trend of policies for balanced development of urban and rural education in eastern, central and western China from the perspective of educational equity. According to the current policies, the main reasons leading to the education gap in eastern, central and western China are discussed. It also clarifies the existing problems of relevant policies, and conducts research and analysis on the future development, aiming to effectively improve the school-running situation in poor and backward areas and to truly realize education for all citizens.
\end{abstract}

Keywords: Educational Equity; Urban and Rural Education; Balance in Education; Educational Gap

\section{Introduction}

Balance in urban-rural education is now an important project in China. In the past period, a large number of policies and rigid development indicators have been put forward for the balanced development of urban and rural education, which has achieved some success. However, there are still many problems to be solved. For example, there are significant differences in education resources and teachers' salaries, seriously hindering the coordinated development of urban and rural education. Efforts are needed for educational equity. From this perspective, to solve the problems, it requires comprehensive management of various interests, together with perfecting relevant policies and systems for creating a good development environment. Then it is various regions and schools to implement the policies. As for the national and local governments, they should give full play to overall planning, strengthen policy support, and fully implement policies for balanced development of education.

\section{Current situation of the policies}

Laws, regulations and policy systems are the key foundation for the balanced development of education in eastern, central and western China, and are also the core for comprehensively promoting the administration of schools and education according to law. From the perspective of educational equity, it is better to advance the idea and promote development later, and the relevant government departments must fully realize their own responsibilities and obligations, and take the initiative to devote to the cause of balance in education, so as to ensure the systematic and stable development of the education in eastern, central and western China. In 2010, the Outline of the National Medium-

Copyright (C) 2020 Jianwei Yang

doi: 10.18282/le.v9i6.1347

This is an open-access article distributed under the terms of the Creative Commons Attribution Non-Commercial License

(http://creativecommons.org/licenses/by-nc/4.0/), which permits unrestricted non-commercial use, distribution, and reproduction in any medium, provided the original work is properly cited. 
and Long-Term Educational Reform and Development Plan (2010-2020) was officially promulgated and implemented, in which the realization of educational equity was regarded as the basic policy.

The current national policy system is mainly to intensify the support for disadvantaged groups and rationally allocate educational and teaching resources, and the overall policy is inclined to rural areas, remote and poverty-stricken areas and ethnic areas, thus effectively narrowing the educational gap. According to the investigation and evaluation results of education in the eastern, central and western regions, the education development in the central region is relatively balanced, while that in the western and eastern regions is with large difference. In terms of the overall development trend, the balanced development of the eastern region is relatively stable, with the policies and measures being actively implemented. However, there is a certain downward trend in the central and western regions. The former is because it has already reached a certain development level and needs to develop in a more targeted direction. The latter has the most serious downward trend, with many loopholes in policies and measures. Influenced by various factors, the balanced development of education in the western regions is greatly hindered.

\section{Analysis of the policies}

\subsection{Policies of school-running conditions}

The conditions for running a school directly depend on the teaching quality and students' quality, and are also the basis for realizing the balanced development of education. Generally speaking, there is an obvious gap between urban and rural areas in the conditions of running schools in the eastern, central and western regions. According to statistics, the per capita number of books in 2019 in ordinary rural primary schools was 30, exceeding that in urban areas which was 22 . This is because the national policy on reading books has been successfully implemented. However, the differences between urban and rural areas in other school-running conditions are quite obvious, which is an urgent problem that needs to be solved in the future ${ }^{[1]}$. These differences lie in the following aspects, namely teaching equipment assets, gymnasiums, multimedia classrooms, and teaching computers. Taking the gymnasium as an example, the per capita gymnasium area of primary schools in eastern urban areas is $0.37 \mathrm{~m}^{2}$, which is 12 times that in rural areas $\left(0.03 \mathrm{~m}^{2}\right)$. By contrast, the figures in western urban areas and rural areas are $0.06 \mathrm{~m}^{2}$ and $0.003 \mathrm{~m}^{2}$ respectively, that is to say the former is 20 times that of the latter. In addition, a comparative analysis of the teaching staff in Jiangsu Province in the east and Guizhou Province in the west was conducted. Figure 1 shows that there is still a big gap in the schoolrunning conditions, and the gap in economically developed areas is smaller than that in economically underdeveloped areas.

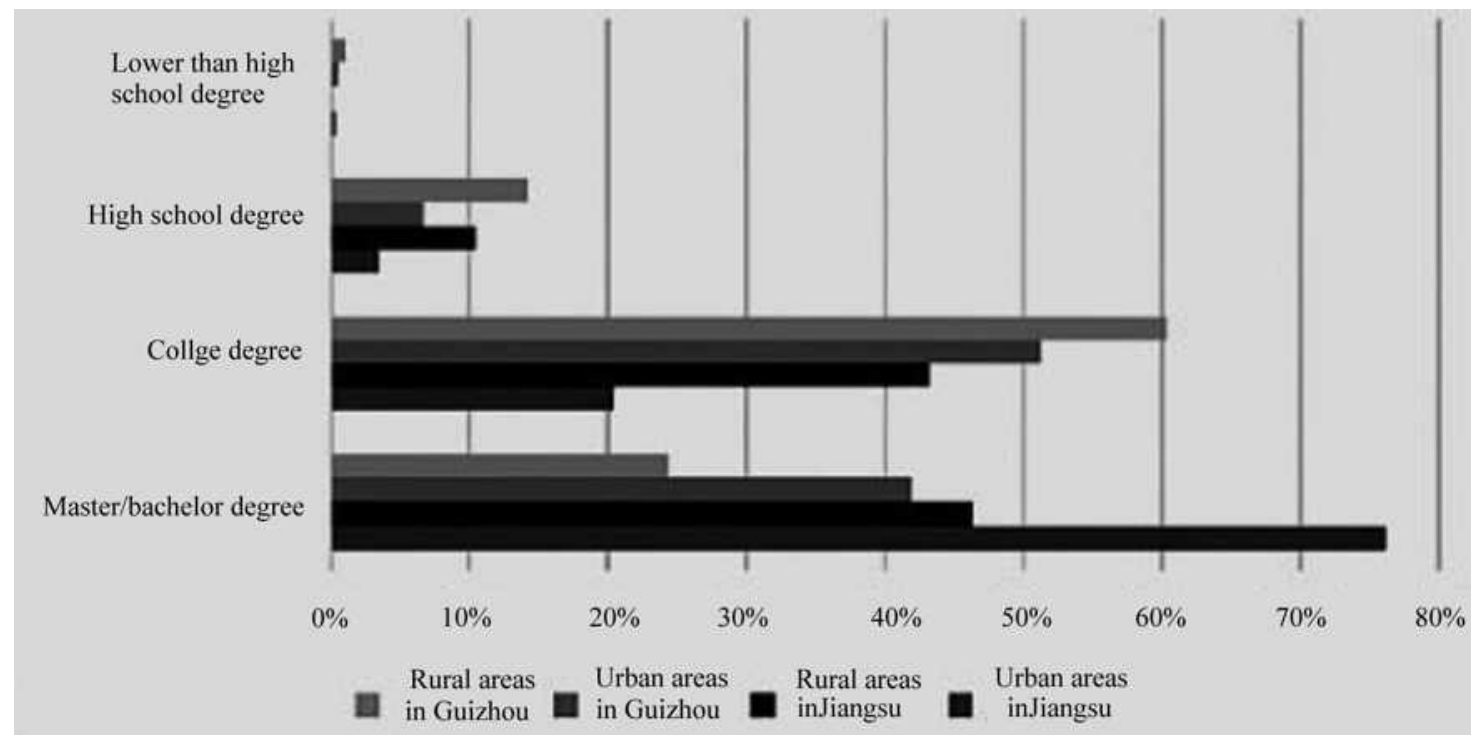

Figure 1. Comparison of the teaching staff between urban and rural areas 


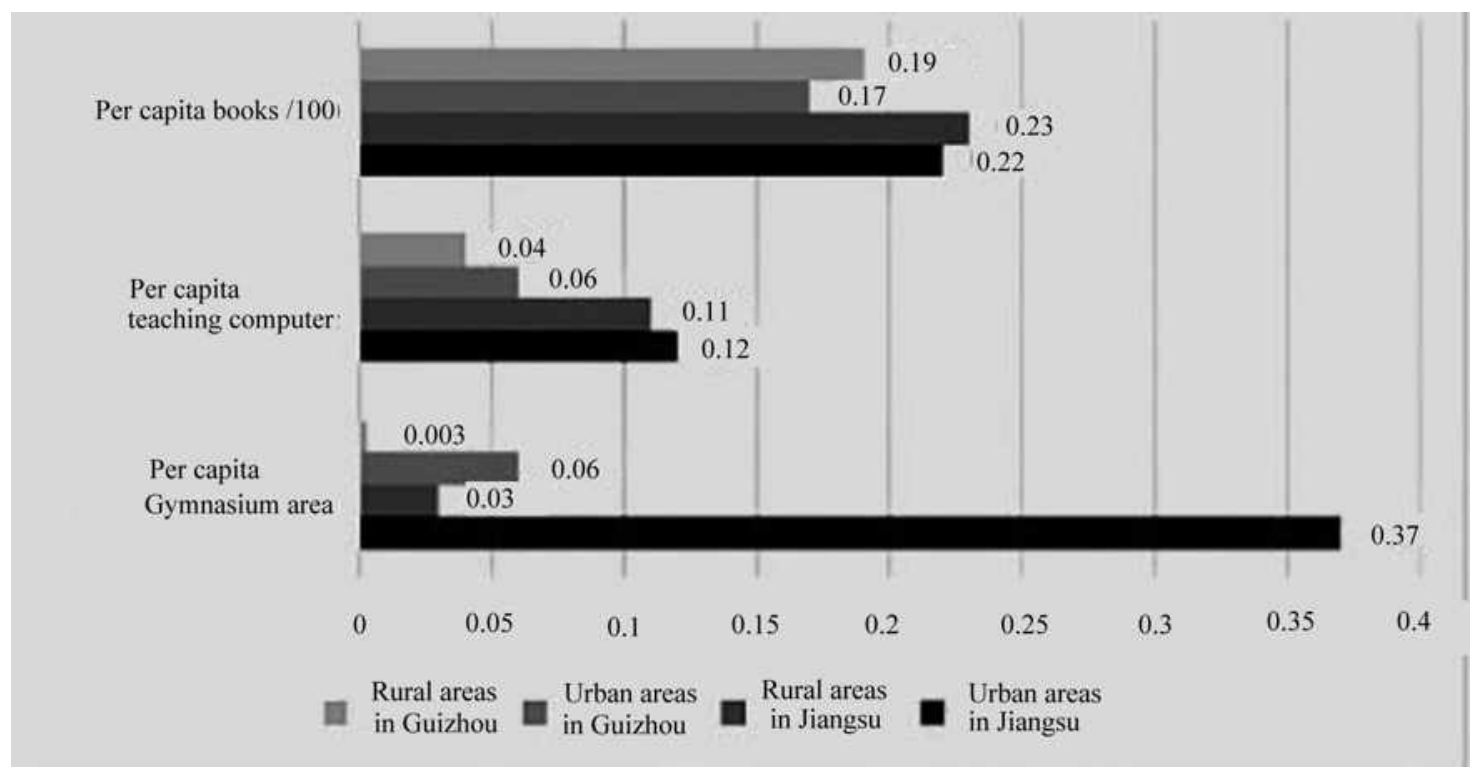

Figure 2. Comparison of school-running conditions between urban and rural areas.

The policy of "two exemptions and one subsidy" refers to "exempting students from incidental fees and textbook fees, and gradually subsidizing boarding students' living expenses". As one of the basic policies to improve the school-running conditions, it has played a certain role in narrowing the gaps between urban and rural areas. Since the implementation of this policy in 2001, it has been funded by the central government to provide financial assistance for students from poor families in compulsory education in rural areas. Among them, textbooks provided free of charge are aimed at 21 key poverty alleviation counties in China and rural students from poor families in compulsory education except children of poverty-stricken rural people. This policy has covered the whole nation in 2017, and is constantly being adjusted according to regional conditions. According to statistics, the policy of "two exemptions and one subsidy" has granted living subsidies to 16.04 million boarding students from urban and rural families with financial difficulties in China, with a subsidy amount of 17.91 billion yuan, of which $90 \%$ is provided for the central and western regions ${ }^{[2]}$. The implementation of this policy has effectively improved the running conditions of backward schools, enabling more students to get access to education.

\subsection{Policies of education expenditure}

Education expenditure is the premise of running a school, not only determining the final quality of education and teaching activities, but also directly affecting the level of running a school. Generally, the current investment of education expenditure in China is relatively low, and there are some gaps between urban and rural education in the eastern, central and western regions, especially in economically underdeveloped areas. From the average level over the whole nation, the investment in education in rural areas is less. The lack of education expenditure has always hindered the development of education in rural areas. The fundamental reason lies in the low total investment of national education expenditure, which leads to the unbalanced allocation of educational resources. In horizontal comparison, the world average of education expenditure in GDP in 2018 was 4.9\%, 5.1\% in developed countries and $4.1 \%$ in underdeveloped countries. The figure in China was $4.31 \%$, indicating a great gap with the world level. In vertical comparison, although the investment in education has increased year by year, the national public budget per capita is 2541.83 yuan, which is 139.74 yuan higher than that in rural areas, directly showing the gap between urban and rural areas. In addition, the investment of educational expenditure in the eastern and central regions is obviously higher than that in the western regions. Taking Guizhou Province-a well-developed province in the western region-as an example, the per capita education budget of rural primary schools is only 2,366.69 yuan, 54.67\% of that in Jiangsu Province in the eastern region. Although the present per capita education expenditure in rural areas over the whole nation can be slightly higher than the average level regardless of economic level, there are still problems in some areas with low economic level, especially in the western region ${ }^{[3]}$.

\subsection{Policies of teaching staff}


As the main body of education and teaching activities, teachers directly determine the quality of education, and are the key to realize the balanced development of education. Nationally, there was still an obvious gap in the academic qualifications of full-time teachers between urban and rural areas in 2019. According to the incomplete investigation and statistical analysis, teachers in urban areas are generally with bachelor degree, while those in rural areas are mainly with college degree. Taking primary school teachers as an example, the proportion of teachers with master degree in urban primary schools was $2.59 \%$ in 2019 , higher than that in rural areas $(0.14 \%)$. And the proportion of teachers with bachelor degree was similar. The proportion of teachers with college degree in rural primary schools was $50 \%$ or even more, while that in urban primary schools was only $35 \%$. The faculty and staff with high school degree or below in rural primary schools accounted for $16.41 \%$, which was 4.7 times that in urban primary schools. The above data show that the problem of uneven distribution of full-time teachers with different academic qualifications between urban and rural schools is obvious, especially that the proportion of highly educated teachers in urban areas is much higher than that in rural areas. For instance, in Guizhou Province, primary school teachers with master degree and bachelor degree in urban areas was $41.95 \%$, which was 1.7 times that in rural areas $(24.35 \%)$. In comparison, the figures in Jiangsu Province were $76.09 \%$ in urban areas and $46.32 \%$ in rural areas, which was 1.6 times. It indicates that there is an obvious gap between urban and rural areas in the eastern, central and western regions, while the gap is greater in economically underdeveloped areas. According to the analysis of the provinces, the academic qualifications of full-time teachers in Jiangsu Province are generally higher than those in Guizhou Province. 61.04\% of the former is with bachelor degree, while $58.4 \%$ of the latter is with college degree and only $30.78 \%$ is with bachelor degree. Therefore, it is necessary for some underdeveloped areas in the eastern, central and western regions to deal with the current situation of "low level in general" ${ }^{,[4]}$.

\section{Improvement of the policies}

\subsection{Policies of school-running conditions}

Science and technology are the primary productive forces, and education is the foundation of the development of science and technology. Education investment can ensure the stable and effective implementation of urban and rural education and teaching activities. Therefore, the Chinese government needs to formulate relevant fiscal policies in the future, increase investment in education, so as to enable economically underdeveloped areas to develop in an all-round way. It is also necessary to improve teaching equipment and teaching conditions, providing material guarantee for the balanced development of education and teaching activities. On the basis of increasing investment in education, the relevant fair mechanism of allocation of educational resources should be applied, such as appropriately reducing the economic investment in economically developed areas and investing more educational expenditure in areas in need, so as to gradually narrow the educational gaps between urban and rural areas and effectively change the status quo. First of all, certain requirements for the management mechanism of education expenditure in rural areas should be put forward by the national government. The education and teaching departments of various regions should increase the education investment in rural areas according to their actual conditions, thus effectively change the current situation of education. Secondly, China needs to rationally expand the allocation of teaching resources to the central and western regions. Affected by various factors, it is difficult to eliminate the differences in economic level, which can be reduced by supporting and helping underdeveloped areas. Finally, the educational resources sharing policy should be established. The educational resources sharing can be achieved with the help of Internet technology, so as to narrow the gap between urban and rural education development.

\subsection{Policies of education supervision}

Although the balance development of urban and rural education in the eastern, central and western regions has been achieved to a certain degree, there are still some problems in the actual implementation of relevant policies and measures, some of which have not reached their original goals and fully played their roles. Generally speaking, there is still an obvious gap between urban and rural education in eastern, central and western China, especially between developed and underdeveloped areas. To continuously promote education equity and balanced development and to reach 
the same educational level in eastern, central and western regions, it is necessary to further improve the corresponding policies, realize the scientific development of educational layout and planning, and perfect the relevant supporting policies, so that the education and teaching activities in various regions of the country can be coordinated. Equity is relative, and the problem of education equity will exist for a long time. Therefore, in the actual development process, supervision of relevant government departments, schools and other educational institutions should be strengthened. Moreover, corruption should be strictly resisted, so that every educational fund can be applied to teaching activities.

\section{Conclusion}

To sum up, balanced development, as a brand-new idea of educational development, is of great significance in promoting education equity and is the foundation of building a harmonious society. However, the government hasn't fully played the macro-control role in eastern, central and western regions, which are affected by natural conditions, historical reasons, economic development and other factors. Therefore, the relevant targeted compensatory education policies need to be further improved.

\section{Reference}

1. Fan H. Assessment and trend prediction of the balanced development of compulsory education in eastern, central and western China (in Chinese). Journal of Hubei University of Science 2019; 39(3): 98-103.

2. Kan L. Current situation, causes and countermeasures of urban and rural educational resources from the perspective of educational equity (in Chinese). Journal of Yanbian Education College 2018; 32(1): 41-44.

3. Jiao W, Jiao L. Research on harmonizing the development of compulsory education school distribution with the newtyped urbanization - based on the investigation of some rural schools in western China. Modern Education Science 2019; 469(4): 24-30.

4. Zhang Q. Differences and countermeasures of urban and rural education development from the perspective of educational equity (in Chinese). Journal of Jiangxi Vocational and Technical College of Electricity 2018; 31(7): 140$141+143$. 